COMMUNICATIONS IN

ANALYSIS AND GEOMETRY

Volume 13, Number 5, 981-998, 2005

\title{
Subsets of Grassmannians Preserved by Mean Curvature Flows
}

\begin{abstract}
Mu-TaO WANG
Let $M=\Sigma_{1} \times \Sigma_{2}$ be the product of two compact Riemannian manifolds of dimension $n \geq 2$ and two, respectively. Let $\Sigma$ be the graph of a smooth map $f: \Sigma_{1} \rightarrow \Sigma_{2} ; \Sigma$ is an $n$-dimensional submanifold of $M$. Let $\mathfrak{G}$ be the Grassmannian bundle over $M$ whose fiber at each point is the set of all $n$-dimensional subspaces of the tangent space of $M$. The Gauss map $\gamma: \Sigma \rightarrow \mathfrak{G}$ assigns to each point $x \in \Sigma$ the tangent space of $\Sigma$ at $x$. This article considers the mean curvature flow of $\Sigma$ in $M$. When $\Sigma_{1}$ and $\Sigma_{2}$ are of the same non-negative curvature, we show a sub-bundle $\mathfrak{S}$ of the Grassmannian bundle is preserved along the flow, i.e. if the Gauss map of the initial submanifold $\Sigma$ lies in $\mathfrak{S}$, then the Gauss map of $\Sigma_{t}$ at any later time $t$ remains in $\mathfrak{S}$. We also show that under this initial condition, the mean curvature flow remains a graph, exists for all time and converges to the graph of a constant map at infinity. As an application, we show that if $f$ is any map from $S^{n}$ to $S^{2}$ and if at each point, the restriction of $d f$ to any two dimensional subspace is area decreasing, then $f$ is homotopic to a constant map.
\end{abstract}

\section{Introduction.}

The maximum principle has proved to be a powerful tool in non-linear partial differential equations. In particular, the maximum principle of parabolic systems for tensors developed by Hamilton [5] plays an important role in the study of geometric evolution equations. The guiding principle is the following: an invariant convex subset in the space of curvature tensors preserved by the associated ordinary differential equations is preserved by the parabolic partial differential equations. This has been applied to the study of Ricci flow and curvature flow of hypersurfaces. We apply this idea to higher codimension mean curvature flows.

Let $M=\Sigma_{1} \times \Sigma_{2}$ be the product of two compact Riemannian manifolds of dimension $n$ and $m$ respectively and $\Sigma$ be the graph of a smooth map $f: \Sigma_{1} \rightarrow \Sigma_{2}$. In [14], [15], and [16], we prove long time existence and convergence theorems for the mean curvature flow of $\Sigma$ assuming conditions 
in terms of the restriction of parallel calibrating forms to $\Sigma$. These are essentially conditions on the tangent spaces of $\Sigma$. In this paper, we take on the study of the Gauss map of $\Sigma$. Let $\mathfrak{G}$ be the Grassmannian bundle over $M$ whose fiber at each point is the set of all $n$-dimensional subspaces of the tangent space of $M$. The Gauss map $\gamma: \Sigma \rightarrow \mathfrak{G}$ assigns to each point $x \in \Sigma$ the tangent space of $\Sigma$ at $x$. The tangent space of $M$ at $x$ splits as $T_{\pi_{1}(x)} \Sigma_{1} \times T_{\pi_{2}(x)} \Sigma_{2}$. Let $\mathfrak{G}^{\prime} \subset \mathfrak{G}$ be the sub-bundle consisting of the graphs of linear transformations from $T_{\pi_{1}(x)} \Sigma_{1}$ to $T_{\pi_{2}(x)} \Sigma_{2}$. We show there exists a sub-bundle $\mathfrak{S} \subset \mathfrak{G}^{\prime}$ that is preserved along the mean curvature flow.

Theorem A. Let $M=\Sigma_{1} \times \Sigma_{2}$ be the product of two compact flat Riemannian manifolds and suppose $\Sigma_{2}$ is two-dimensional. If the Gauss map of a compact oriented submanifold $\Sigma$ of $M$ lies in $\mathfrak{S}$, then along the mean curvature flow the Gauss map of $\Sigma_{t}$ remains in $\mathfrak{S}$. The flow exists smoothly for all time and converges to a totally geodesic submanifold.

This condition in particular implies $\Sigma_{t}$ is the graph of a map $f_{t}$. The sub-bundle $\mathfrak{S}$ is best described in terms of $f_{t}$. In fact, if we denote the singular values of $f_{t}$ by $\lambda_{1}$ and $\lambda_{2}$, then the Gauss map of $\Sigma_{t}$ lies in $\mathfrak{S}$ if and only if $\left|\lambda_{1} \lambda_{2}\right|<1$.

When $\Sigma_{1}$ is of positive curvature, we prove the following.

Theorem B. Let $M=S^{n}\left(k_{1}\right) \times \Sigma_{2}$ be the product of an $n$-sphere of curvature $k_{1}>0$ and a two-dimensional compact Riemannian manifold $\Sigma_{2}$ of constant curvature $k_{2}$ and $k_{1} \geq\left|k_{2}\right|$. If the Gauss map of a compact oriented submanifold $\Sigma$ of $M$ lies in $\mathfrak{S}$, then along the mean curvature flow, the Gauss map of $\Sigma_{t}$ remains in $\mathfrak{S}$. The flow exists smoothly for all time and converges to a totally geodesic submanifold.

We noted the assumption on the Gauss map of $\Sigma$ implies $\Sigma$ is a graph over $S^{n}\left(k_{1}\right)$.

Theorem A and B are proved by calculating the evolution equations of the Gauss map and applying the maximum principle. The prototype is the following equation in the hypersurface case

$$
\frac{d}{d t} N=\Delta N+|A|^{2} N
$$

where $N$ denotes the unit normal vector and $|A|^{2}$ is the norm of the second fundamental form. If we take the inner product of $N$ with a constant vector $\nu$, it is not hard to see that $\min _{\Sigma_{t}}\langle N, \nu\rangle$ is non-decreasing in time. This is one of the key observations in [2] and [3] where the mean curvature flow of entire 
graphs of codimension one was studied. In the case of hypersurfaces, $N$ contains all the information of the Gauss map while in higher codimension, a whole parabolic system is needed in order to describe the evolution of the Gauss map.

The following is an application to higher homotopy groups of $S^{2}$.

Corollary. If $f$ is any map from $S^{n}$ to $S^{2}, n \geq 2$ and if at each point, the restriction of $d f$ to any two dimensional subspace is area decreasing, then $f$ is homotopic to a constant map along the mean curvature flow.

When $n=2$, this is the same as saying the Jacobian of $f$ is less than 1 . In this case, $f$ is of degree 0 and thus homotopic to a constant map. This homotopy can be realized through the mean curvature flow as was proved in [14]. As a contrast, the standard Hopf map from $S^{3}$ to $S^{2}$ has $\left|\lambda_{1} \lambda_{2}\right|=4$.

The author is indebted to Professor D. H. Phong and Professor S.-T. Yau for their constant encouragement and unending support. He has benefitted greatly from the conversation with Professor R. Hamilton and Professor M-P Tsui. The author is partially supported by an NSF grant.

\section{Analysis of the Grassmannian bundle.}

Let us first describe the sub-bundle $\mathfrak{S}$. Let $V_{1}$ be an $n$-dimensional inner product space and $V_{2}$ a two-dimensional inner product space. Let $G(n, n+2)$ be the Grassmannian of all $n$-dimensional subspaces of $V_{1} \times V_{2}$. Let $G^{\prime} \subset$ $G(n, n+2)$ be the set of all $n$-dimensional subspaces that can be written as graphs over $V_{1}$. For any $P \in G^{\prime}, P$ is the graph of a linear transformation $\mathfrak{p}: V_{1} \rightarrow V_{2}$. Then $(\mathfrak{p})^{T} \mathfrak{p}$ is a self-adjoint map from $V_{1}$ to $V_{1}$ and has $n$ eigenvalues, $n-2$ of them vanish. The eigenvalues are denoted by $\left\{\lambda_{1}^{2}, \lambda_{2}^{2}\right\}$. $\lambda_{1}$ and $\lambda_{2}$ are the singular values of $\mathfrak{p}$. We now define $S$ :

$$
S=\left\{P \in G^{\prime}|1-| \lambda_{1} \lambda_{2} \mid>0\right\} .
$$

This is equivalent to saying $\mathfrak{p}$ is area decreasing on any two dimensional subspace of $V_{1}$.

Now let $M$ be the product of two Riemannian manifolds $\Sigma_{1} \times \Sigma_{2}$ of dimension $n$ and 2 respectively. Let $\mathfrak{G}$ be the Grassmannian bundle on $M$ whose fibers are isomorphic to $G(n, n+2)$. At each point $x, T_{x} M$ splits as the product of $T_{\pi_{1}(x)} \Sigma_{1}$ and $T_{\pi_{2}(x)} \Sigma_{2}$. The Riemannian structures on $T_{\pi_{1}(x)} \Sigma_{1}=V_{1}$ and $T_{\pi_{2}(x)} \Sigma_{2}=V_{2}$ defines the subset $S$ of the fiber of $\mathfrak{G}$ at $x$.

Definition 2.1. $\mathfrak{S}$ is the sub-bundle of the Grassmannian bundle $\mathfrak{G}$ whose fiber at each point consists of $S$. 
Let $\Sigma$ be the graph of a smooth map $f: \Sigma_{1} \rightarrow \Sigma_{2}$. $T_{x} \Sigma$ is the graph of the differential of $f$ at $x, d f: T_{\pi_{1}(x)} \Sigma_{1} \rightarrow T_{\pi_{2}(x)} \Sigma_{2}$. Notice that we abuse the notation so that $T_{x} \Sigma, T_{\pi_{1}(x)} \Sigma_{1}$ and $T_{\pi_{2}(x)} \Sigma_{2}$ all denote subspaces of $T_{x} M$. At any point $x$, let $\lambda_{1}, \lambda_{2}$ be the singular values of $d f$. They are well-defined up to a sign. Define

$$
\eta(x)=\frac{1-\left|\lambda_{1} \lambda_{2}\right|}{\sqrt{\left(1+\lambda_{1}^{2}\right)\left(1+\lambda_{2}^{2}\right)}}
$$

$\eta$ is a function on $\Sigma$.

Proposition 2.2. $\eta>0$ on $\Sigma$ if and only if the Gauss map of $\Sigma$ lies in $\mathfrak{S}$.

Later we shall give a characterization of $\eta$ in terms of differential forms on $M$. Any differential form $\Omega$ on a Riemannian manifold can be considered as a function on the Grassmannian bundle $\mathfrak{G}$ of appropriate dimension. The comass of $\Omega$ at $x$ is defined to be the supremum of $\Omega$ on $\mathfrak{G}_{x}$, the fiber of $\mathfrak{G}$ at $x$. This is an important concept in calibrated geometry, see Federer [4] or Harvey-Lawson [6]. Another description of $\mathfrak{S}$ can be given in terms of the comass.

Proposition 2.3. If $\Sigma$ is the graph of $f: \Sigma_{1} \rightarrow \Sigma_{2}$, then the Gauss map of $\Sigma$ lies in $\mathfrak{S}$ if and only if the comass of $f^{*} \Omega_{2}$ is less than one.

Here $\Omega_{2}$ is the volume form on $\Sigma_{2}$ and $f^{*} \Omega_{2}$ is considered as a 2-form on $\Sigma_{1}$. Of course the comass is taken over all two-dimensional subspaces of the tangent space of $\Sigma_{1}$.

\section{Evolution equations of $n$-forms.}

In this section, we calculate the evolution equation of the restriction of an $n$ form to an $n$-dimensional submanifold moving by the mean curvature flow. The case for a parallel form was calculated in [16]. Here, we need to keep track of the terms that involve covariant derivatives of $\Omega$.

We assume $M$ is an $n+m$ dimensional Riemannian manifold with an $n$ form $\Omega$. Let $F: \Sigma \rightarrow M$ be an isometric immersion of an $n$-dimensional submanifold. We shall compute near a point $p \in \Sigma$. We choose arbitrary orthonormal frames $\left\{e_{i}\right\}_{i=1 \cdots n}$ for $T \Sigma$ and $\left\{e_{\alpha}\right\}_{\alpha=n+1, \cdots, n+m}$ for $N \Sigma$. $\nabla^{M}$ denotes the covariant derivative on $M$ and $\nabla^{\Sigma}$ denotes the covariant derivative on $\Sigma$, which is simply the tangent part of $\nabla^{M}$. $\nabla^{M} \Omega$ is the covariant derivative of $\Omega$ on $M$ and $\nabla^{\Sigma} \Omega$ will denote the covariant derivative of the restriction of $\Omega$ to $\Sigma$. 
We first calculate the covariant derivative of the restriction of $\Omega$ on $\Sigma$.

$$
\begin{aligned}
& \left(\nabla_{e_{k}}^{\Sigma} \Omega\right)\left(e_{i_{1}}, \cdots, e_{i_{n}}\right) \\
& =e_{k}\left(\Omega\left(e_{i_{1}}, \cdots, e_{i_{n}}\right)\right)-\Omega\left(\nabla_{e_{k}}^{\Sigma} e_{i_{1}}, \cdots, e_{i_{n}}\right)-\cdots-\Omega\left(e_{i_{1}}, \cdots, \nabla_{e_{k}}^{\Sigma} e_{i_{n}}\right) \\
& =\left(\nabla_{e_{k}}^{M} \Omega\right)\left(e_{i_{1}}, \cdots, e_{i_{n}}\right)+\Omega\left(\nabla_{e_{k}}^{M} e_{i_{1}}-\nabla_{e_{k}}^{\Sigma} e_{i_{1}}, \cdots, e_{i_{n}}\right) \\
& \quad \quad+\cdots+\Omega\left(e_{i_{1}}, \cdots, \nabla_{e_{k}}^{M} e_{i_{n}}-\nabla_{e_{k}}^{\Sigma} e_{i_{n}}\right)
\end{aligned}
$$

This equation can be abbreviated using the second fundamental form of $F, h_{\alpha i j}=\left\langle\nabla_{e_{i}}^{M} e_{j}, e_{\alpha}\right\rangle$. We write $\Omega_{i_{1} \cdots i_{n}, k}$ shorthand for $\left(\nabla_{e_{k}}^{\Sigma} \Omega\right)\left(e_{i_{1}}, \cdots, e_{i_{n}}\right)$.

$$
\Omega_{i_{1} \cdots i_{n}, k}=\left(\nabla_{e_{k}}^{M} \Omega\right)\left(e_{i_{1}}, \cdots, e_{i_{n}}\right)+\Omega_{\alpha i_{2} \cdots i_{n}} h_{\alpha i_{1} k}+\cdots+\Omega_{i_{1} \cdots i_{n-1} \alpha} h_{\alpha i_{n} k}
$$

Likewise, in $\Omega\left(e_{\alpha}, e_{i_{2}}, \cdots, e_{i_{n}}\right), \Omega$ is considered as a section of $(N \Sigma)^{*} \wedge$ $\left(\wedge^{n-1}(T \Sigma)^{*}\right)$

$$
\begin{aligned}
\Omega_{\alpha i_{2} \cdots i_{n}, k}=( & \left.\nabla_{e_{k}}^{M} \Omega\right)\left(e_{\alpha}, e_{i_{1}}, \cdots, e_{i_{n}}\right)-\Omega_{j i_{2} \cdots i_{n}} h_{\alpha j k}+\Omega_{\alpha \beta i_{3} \cdots i_{n}} h_{\beta i_{2} k} \\
& +\cdots+\Omega_{\alpha i_{2} \cdots i_{n-1} \beta} h_{\beta i_{n} k}
\end{aligned}
$$

Now, we calculate the second covariant derivative of the restriction of $\Omega$ on $\Sigma$.

$$
\begin{aligned}
& \left(\nabla_{e_{k}}^{\Sigma} \nabla_{e_{k}}^{\Sigma} \Omega\right)\left(e_{1}, \cdots e_{n}\right) \\
& =e_{k}\left(\left(\nabla_{e_{k}}^{\Sigma} \Omega\right)\left(e_{1}, \cdots e_{n}\right)\right)-\left(\nabla_{e_{k}}^{\Sigma} \Omega\right)\left(\nabla_{e_{k}}^{\Sigma} e_{1}, \cdots, e_{n}\right) \\
& \quad-\cdots-\left(\nabla_{e_{k}}^{\Sigma} \Omega\right)\left(e_{1}, \cdots, \nabla_{e_{k}}^{\Sigma} e_{n}\right)
\end{aligned}
$$

The term $\left(\nabla_{e_{k}}^{\Sigma} \Omega\right)\left(\nabla_{e_{k}}^{\Sigma} e_{1}, \cdots, e_{n}\right)$ equals zero because $\nabla_{e_{k}}^{\Sigma} e_{1}$ is a tangent vector perpendicular to $e_{1}$ and thus a linear combination of $e_{2}, \cdots, e_{n}$. Likewise, other similar terms vanish.

$$
\begin{aligned}
\left(\nabla_{e_{k}}^{\Sigma} \nabla_{e_{k}}^{\Sigma} \Omega\right)\left(e_{1}, \cdots e_{n}\right) & =e_{k}\left[\left(\nabla_{e_{k}}^{M} \Omega\right)\left(e_{1}, \cdots, e_{n}\right)+\Omega_{\alpha 2 \cdots n} h_{\alpha 1 k}+\cdots+\Omega_{1 \cdots n-1 \alpha} h_{\alpha n k}\right] \\
= & \left(\nabla_{e_{k}}^{M} \nabla_{e_{k}}^{M} \Omega\right)\left(e_{1}, \cdots, e_{n}\right)+\left(\nabla_{e_{k}}^{M} \Omega\right)\left(\nabla_{e_{k}}^{M} e_{1}, \cdots, e_{n}\right) \\
& +\cdots+\left(\nabla_{e_{k}}^{M} \Omega\right)\left(e_{1}, \cdots, \nabla_{e_{k}}^{M} e_{n}\right) \\
& +\cdots+\Omega_{1 \cdots n-1 \alpha, k} h_{\alpha n k} \\
& +\Omega_{\alpha 2 \cdots n, k} h_{\alpha 1 k}+\cdots+\Omega_{1 \cdots n} \\
& +\Omega_{\alpha 2 \cdots n} h_{\alpha 1 k, k}+\cdots+\Omega_{1 \cdots n-1 \alpha} h_{\alpha n k, k}
\end{aligned}
$$

Now, $\nabla_{e_{k}}^{M} e_{i}=h_{\alpha i k} e_{\alpha}+\nabla_{e_{k}}^{\Sigma} e_{i}$ and $\left(\nabla_{e_{k}}^{M} \Omega\right)\left(\nabla_{e_{k}}^{\Sigma} e_{1}, \cdots, e_{n}\right)=0$. 
Therefore,

$$
\begin{aligned}
\Omega_{1 \cdots n, k k}= & \left(\nabla_{e_{k}}^{M} \nabla_{e_{k}}^{M} \Omega\right)\left(e_{1}, \cdots, e_{n}\right) \\
& +\left(\nabla_{e_{k}}^{M} \Omega\right)\left(e_{\alpha}, \cdots, e_{n}\right) h_{\alpha 1 k}+\cdots+\left(\nabla_{e_{k}}^{M} \Omega\right)\left(e_{1}, \cdots, e_{\alpha}\right) h_{\alpha n k} \\
& +\Omega_{\alpha 2 \cdots n, k} h_{\alpha 1 k}+\cdots+\Omega_{1 \cdots n-1 \alpha, k} h_{\alpha n k} \\
& +\Omega_{\alpha 2 \cdots n} h_{\alpha 1 k, k}+\cdots+\Omega_{1 \cdots n-1 \alpha} h_{\alpha n k, k}
\end{aligned}
$$

Plug equation (3.2) into (3.4) and apply the Codazzi equation $h_{\alpha k i, k}=$ $h_{\alpha, i}+R_{\alpha k k i}$ where $R$ is the curvature operator of $M, h_{\alpha}$ is the component of the mean curvature vector $H=h_{\alpha} e_{\alpha}$, and $h_{\alpha, i}$ is the component of the covariant derivative of $H$ as a section of the normal bundle, i.e. $\left(\nabla_{e_{i}} H\right)^{N}=$ $h_{\alpha, i} e_{\alpha}$.

$$
\begin{aligned}
\left(\Delta^{\Sigma} \Omega\right)_{1 \cdots n}= & -\Omega_{12 \cdots n} \sum_{\alpha, k}\left(h_{\alpha 1 k}^{2}+\cdots+h_{\alpha n k}^{2}\right) \\
& +2 \sum_{\alpha, \beta, k}\left[\Omega_{\alpha \beta 3 \cdots n} h_{\alpha 1 k} h_{\beta 2 k}+\Omega_{\alpha 2 \beta \cdots n} h_{\alpha 1 k} h_{\beta 3 k}\right. \\
& \left.+\cdots+\Omega_{1 \cdots(n-2) \alpha \beta} h_{\alpha(n-1) k} h_{\beta n k}\right] \\
& +\sum_{\alpha, k} \Omega_{\alpha 2 \cdots n} h_{\alpha, 1}+\cdots+\Omega_{1 \cdots(n-1) \alpha} h_{\alpha, n} \\
& +\sum_{\alpha, k} \Omega_{\alpha 2 \cdots n} R_{\alpha k k 1}+\cdots+\Omega_{1 \cdots(n-1) \alpha} R_{\alpha k k n} \\
& +\left(\nabla_{e_{k}}^{M} \nabla_{e_{k}}^{M} \Omega\right)\left(e_{1}, \cdots, e_{n}\right) \\
& +2\left(\nabla_{e_{k}}^{M} \Omega\right)\left(e_{\alpha}, \cdots, e_{n}\right) h_{\alpha 1 k}+\cdots+2\left(\nabla_{e_{k}}^{M} \Omega\right)\left(e_{1}, \cdots, e_{\alpha}\right) h_{\alpha n k}
\end{aligned}
$$

We notice that $\left(\Delta^{\Sigma} \Omega\right)_{1 \cdots n}=\Delta\left(\Omega\left(e_{1}, \cdots, e_{n}\right)\right)$, where the $\Delta$ on the righthand side is the Laplacian of functions on $\Sigma$.

The terms in the brackets are formed in the following way. Choose two different indexes from 1 to $n$, replace the smaller one by $\alpha$ and the larger one by $\beta$. There are a total of $\frac{n(n-1)}{2}$ such terms.

Now, we consider when $\Sigma=\Sigma_{t}$ is a time slice of a mean curvature flow in $M$ by $\frac{d}{d t} F_{t}=H_{t}$. Notice that, here we require the velocity vector is in the normal direction. We can extend $e_{1}, \cdots, e_{n}$ to a local coordinate system 


$$
\begin{aligned}
& \left\{\partial_{i}=\frac{\partial}{\partial x^{i}}\right\} \text { on } \Sigma \text {, then } \\
& \qquad \begin{aligned}
\frac{d}{d t} \Omega & \left(\partial_{1}, \cdots, \partial_{n}\right) \\
= & \left(\nabla_{H}^{M} \Omega\right)\left(\partial_{1}, \cdots, \partial_{n}\right)+\Omega\left(\left(\nabla_{\partial_{1}} H\right)^{N}, \partial_{2}, \cdots, \partial_{n}\right) \\
& +\cdots+\Omega\left(\partial_{1}, \partial_{2}, \cdots,\left(\nabla_{\partial_{n}} H\right)^{N}\right) \\
& +\Omega\left(\left(\nabla_{\partial_{1}} H\right)^{T}, \partial_{2}, \cdots, \partial_{n}\right)+\cdots+\Omega\left(\partial_{1}, \partial_{2}, \cdots,\left(\nabla_{\partial_{n}} H\right)^{T}\right)
\end{aligned}
\end{aligned}
$$

Since $\frac{d}{d t} g_{i j}=\left\langle\left(\nabla_{\partial_{i}} H\right)^{T}, \partial_{j}\right\rangle$, if we choose an orthonormal frame and evolve the frame with respect to time so that it remains orthonormal, the terms in the last line vanish. Denote

$$
* \Omega=\frac{1}{\sqrt{\operatorname{det} g_{i j}}} \Omega\left(\partial_{1}, \cdots, \partial_{n}\right)
$$

then $* \Omega$ satisfies

$$
\frac{d}{d t} * \Omega=*\left(\nabla_{H}^{M} \Omega\right)+\Omega_{\alpha 2 \cdots n} h_{\alpha, 1}+\cdots+\Omega_{1 \cdots(n-1) \alpha} h_{\alpha, n}
$$

Combining this with equation (3.5), we get the parabolic equation satisfied by $* \Omega$.

Proposition 3.1. Suppose $\Sigma_{t}$ is a time slice of an $n$-dimensional mean curvature flow in $M$ and $\Omega$ is an n-form on $M$. For any point $p \in \Sigma_{t}$, let $\left\{e_{1}, \cdots, e_{n}\right\}$ be an orthonormal frame of $T \Sigma_{t}$ near $p$ and $\left\{e_{n+1}, \cdots, e_{n+m}\right\}$ be an orthonormal frame of the normal bundle of $\Sigma_{t}$ near $p$. Then $* \Omega=$ $\Omega\left(e_{1}, \cdots, e_{n}\right)$ satisfies

$$
\begin{aligned}
\frac{d}{d t} * \Omega= & \Delta * \Omega+* \Omega\left(\sum_{\alpha, i, k} h_{\alpha i k}^{2}\right) \\
& -2 \sum_{\alpha, \beta, k}\left[\Omega_{\alpha \beta 3 \cdots n} h_{\alpha 1 k} h_{\beta 2 k}+\Omega_{\alpha 2 \beta \cdots n} h_{\alpha 1 k} h_{\beta 3 k}\right. \\
& \left.+\cdots+\Omega_{1 \cdots(n-2) \alpha \beta} h_{\alpha(n-1) k} h_{\beta n k}\right] \\
& -\sum_{\alpha, k}\left[\Omega_{\alpha 2 \cdots n} R_{\alpha k k 1}+\cdots+\Omega_{1 \cdots(n-1) \alpha} R_{\alpha k k n}\right] \\
& +*\left(\nabla_{H}^{M} \Omega\right)-\left(\nabla_{e_{k}}^{M} \nabla_{e_{k}}^{M} \Omega\right)\left(e_{1}, \cdots, e_{n}\right) \\
& -2\left(\nabla_{e_{k}}^{M} \Omega\right)\left(e_{\alpha}, \cdots, e_{n}\right) h_{\alpha 1 k}-\cdots-2\left(\nabla_{e_{k}}^{M} \Omega\right)\left(e_{1}, \cdots, e_{\alpha}\right) h_{\alpha n k}
\end{aligned}
$$

where $\Delta$ denotes the time-dependent Laplacian on $\Sigma_{t}$. 


\section{Proof of Theorems.}

Let us prove Theorem A now. We recall the statement.

Theorem A. Let $M=\Sigma_{1} \times \Sigma_{2}$ be the product of two compact flat Riemannian manifolds of dimension $n$ and 2 , respectively. If the Gauss map of a compact oriented submanifold $\Sigma$ of $M$ lies in $\mathfrak{S}$, then along the mean curvature flow the Gauss map of $\Sigma_{t}$ remains in $\mathfrak{S}$. The flow exists smoothly for all time and converges to a totally geodesic submanifold.

Proof. Let $\Sigma_{t}$ be the mean curvature flow of $\Sigma$ given by a family of immersions $F: \Sigma \times[0, T) \rightarrow M$. In the following calculation, it is useful to consider the total space of the mean curvature flow as $\Sigma \times[0, T)$. At each instant $t, \Sigma$ is equipped with the induced metric by $F_{t}$. All geometric quantities defined on the image of $F(\cdot, t)$ are considered as defined on $\Sigma$.

Let $\Omega_{1}$ and $\Omega_{2}$ be the volume form of $\Sigma_{1}$ and $\Sigma_{2}$ respectively. They can be considered as parallel forms on $M$. Suppose initially the image of the Gauss map of $\Sigma$ is in $\mathfrak{S}$. We may assume $\Sigma$ is the graph of a map $f: \Sigma_{1} \rightarrow \Sigma_{2}$. This implies $\eta_{1}=* \Omega_{1}>0$ and $\eta>0$ on $\Sigma$ at $t=0$ by Proposition 2.2.

We shall characterize $\eta$ in terms of differential forms. Consider $\Xi$ the collection of $n$ forms on $M$ of the following type.

$$
\begin{aligned}
& \Xi=\left\{\Omega=\Omega_{1}-\Omega_{2} \wedge \omega \mid \omega \text { is any parallel simple }(n-2)\right. \text { form } \\
& \left.\quad \text { of comass one on } \Sigma_{1}\right\}
\end{aligned}
$$

At any point $x$, by Singular Value Decomposition, we can take an orthonormal basis $\left\{a_{i}\right\}_{i=1 \cdots n}$ for $T_{\pi_{1}(x)} \Sigma_{1}$ and $\left\{a_{\alpha}\right\}_{\alpha=n+1, n+2}$ for $T_{\pi_{2}(x)} \Sigma_{2}$ so that $d f\left(a_{i}\right)=\lambda_{i} a_{n+i}, a_{1}^{*} \wedge \cdots \wedge a_{n}^{*}$ is the volume form of $T_{\pi_{1}(x)} \Sigma_{1}$ and $a_{n+1}^{*} \wedge a_{n+2}^{*}$ is the volume form for $T_{\pi_{2}(x)} \Sigma_{2}$. Therefore,

$$
\begin{gathered}
\left\{e_{1}=\frac{1}{\sqrt{1+\lambda_{1}^{2}}}\left(a_{1}+\lambda_{1} a_{n+1}\right), e_{2}=\frac{1}{\sqrt{1+\lambda_{2}^{2}}}\left(a_{2}+\lambda_{2} a_{n+2}\right),\right. \\
\left.e_{3}=a_{3}, \cdots, e_{n}=a_{n}\right\}
\end{gathered}
$$

forms an orthonormal basis for $T_{x} \Sigma$ and

$$
\left\{e_{n+1}=\frac{1}{\sqrt{1+\lambda_{1}^{2}}}\left(a_{n+1}-\lambda_{1} a_{1}\right), e_{n+2}=\frac{1}{\sqrt{1+\lambda_{2}^{2}}}\left(a_{n+2}-\lambda_{2} a_{2}\right)\right\}
$$


an orthonormal basis for $N_{x} \Sigma$. Thus,

$$
\begin{aligned}
*\left(\Omega_{1}-\Omega_{2} \wedge \omega\right) & =\left(\Omega_{1}-\Omega_{2} \wedge \omega\right)\left(e_{1}, \cdots, e_{n}\right) \\
& =\frac{1}{\sqrt{\left(1+\lambda_{1}^{2}\right)\left(1+\lambda_{2}^{2}\right)}}\left(1-\lambda_{1} \lambda_{2} \omega\left(a_{3}, \cdots, a_{n}\right)\right)
\end{aligned}
$$

On the other hand

$$
\eta_{1}=\frac{1}{\sqrt{\left(1+\lambda_{1}^{2}\right)\left(1+\lambda_{2}^{2}\right)}}
$$

Recall

$$
\eta=\frac{1-\left|\lambda_{1} \lambda_{2}\right|}{\sqrt{\left(1+\lambda_{1}^{2}\right)\left(1+\lambda_{2}^{2}\right)}}
$$

It is not hard to see

$$
\eta(x)=\min _{\Omega \in \Xi} * \Omega(x)
$$

Suppose at $t=t_{0}$, the image of the Gauss map hits the boundary of $\mathfrak{S}$ for the first time. Therefore, each $\Sigma_{t}, t<t_{0}$ can be written as the graph of $f_{t}: \Sigma_{1} \rightarrow \Sigma_{2}$ and the singular values of $f_{t}$ satisfy $\left|\lambda_{1} \lambda_{2}\right|<1$.

We claim $\Sigma_{t_{0}}$ remains a graph. Indeed, since $\Omega_{1}$ is a parallel form, $\eta_{1}$ satisfies the following equation by equation (3.6).

$$
\frac{d}{d t} \eta_{1}=\Delta \eta_{1}+\eta_{1}\left[\sum_{\alpha, i, k} h_{\alpha i k}^{2}-2 \sum_{k} \lambda_{1} \lambda_{2}\left(h_{n+1,1 k} h_{n+2,2 k}-h_{n+2,1 k} h_{n+1,2 k}\right)\right]
$$

where we use

$$
\begin{aligned}
\Omega_{1}\left(e_{n+1}, e_{n+2}, e_{3}, \cdots, e_{n}\right) & =\frac{\Omega_{1}\left(a_{n+1}-\lambda_{1} a_{1}, a_{n+2}-\lambda_{2} a_{2}, a_{3}, \cdots, a_{n}\right)}{\sqrt{\left(1+\lambda_{1}^{2}\right)\left(1+\lambda_{2}^{2}\right)}} \\
& =\lambda_{1} \lambda_{2} \eta_{1} .
\end{aligned}
$$

Notice this equation is valid at any point $x$. Since $\left|\lambda_{1} \lambda_{2}\right|<1$ for $0 \leq$ $t<t_{0}$, applying the maximum principle to equation (4.4) implies $\min _{\Sigma_{t}} \eta_{1}$ is non-decreasing in $t$ and thus $\eta_{1}>0$ at $t_{0}$.

Now, $\eta$ is well-defined at $t_{0}$. Take any $p$ so that $\eta\left(p, t_{0}\right)=0$, we shall show that $\left.\frac{d}{d t}\right|_{t=t_{0}} \eta \geq 0$ at $p$.

It is clear that $\lambda_{1} \lambda_{2} \neq 0$ at $p$. Otherwise, $\eta_{1}=\eta=0$, a contradiction.

By the previous characterization of $\eta$ and Hamilton's maximum principle [5], we only need to show $\left.\frac{d}{d t}\right|_{t=t_{0}} * \Omega \geq 0$ at the point $p$ for any $\Omega \in \Xi$ such that $* \Omega(p)=\eta(p)$. At $p$, we apply Singular Value Decomposition to get an 
orthonormal basis $\left\{a_{i}\right\}_{i=1, \cdots n}$ for $T_{\pi_{1}(p)} \Sigma_{1}$ as before. Such $\Omega$ is of the form $\Omega_{1}-\Omega_{2} \wedge \omega$ with $\omega\left(a_{3}, \cdots, a_{n}\right)=1$ or $\omega=a_{3}^{*} \wedge \cdots \wedge a_{n}^{*}$ by equation (4.3).

$* \Omega$ satisfies

$$
\begin{aligned}
\frac{d}{d t} * \Omega= & \Delta * \Omega+* \Omega\left(\sum_{\alpha, i, k} h_{\alpha i k}^{2}\right) \\
- & 2 \sum_{\alpha, \beta, k}\left[\Omega_{\alpha \beta 3 \cdots n} h_{\alpha 1 k} h_{\beta 2 k}+\Omega_{\alpha 2 \beta \cdots n} h_{\alpha 1 k} h_{\beta 3 k}\right. \\
& \left.+\cdots+\Omega_{1 \cdots(n-2) \alpha \beta} h_{\alpha(n-1) k} h_{\beta n k}\right] .
\end{aligned}
$$

At this point $p$,

$$
\begin{aligned}
& \left(\Omega_{1}-\Omega_{2} \wedge \omega\right)\left(e_{n+1}, e_{n+2}, e_{i_{1}}, \cdots e_{i_{n-2}}\right) \\
& \quad=\frac{1}{\sqrt{\left(1+\lambda_{1}^{2}\right)\left(1+\lambda_{2}^{2}\right)}}\left(\lambda_{1} \lambda_{2}-1\right) \omega\left(e_{i_{1}}, \cdots, e_{i_{n-2}}\right) .
\end{aligned}
$$

Thus

$$
\frac{d}{d t} * \Omega=\Delta * \Omega+* \Omega\left[|A|^{2}+2\left(h_{n+1,1 k} h_{n+2,2 k}-h_{n+1,2 k} h_{n+2,1 k}\right)\right] .
$$

This can be completed square and we get

$$
\begin{aligned}
\frac{d}{d t} * \Omega= & \Delta * \Omega+* \Omega\left[\sum_{\alpha, 2<i \leq n, k} h_{\alpha i k}^{2}+\sum_{k}\left(h_{n+1,1 k}+h_{n+2,2 k}\right)^{2}\right. \\
& \left.+\sum_{k}\left(h_{n+1,2 k}-h_{n+2,1 k}\right)^{2}\right] .
\end{aligned}
$$

Therefore, $\frac{d}{d t} * \Omega \geq 0$ at $\left(p, t_{0}\right)$. Since this is true for any $\Omega$ that achieves the minimum of $* \Omega$ in $\Xi$, we have $\frac{d}{d t} \eta \geq 0$. Thus, the sub-bundle $\mathfrak{S}$ is preserved along the mean curvature flow.

Now, we prove long time existence and convergence. By a similar argument, we can show if $\min \eta=\delta>0$ at $t=0$, then this is preserved along the flow. This implies in particular,

$$
\left|\lambda_{1} \lambda_{2}\right| \leq 1-\delta
$$

and

$$
\sqrt{\left(1+\lambda_{1}^{2}\right)\left(1+\lambda_{2}^{2}\right)} \leq \frac{1}{\delta}
$$


Since $\left|\lambda_{1} \lambda_{2}\right| \leq 1-\delta$, we have

$$
\frac{d}{d t} * \Omega_{1} \geq \Delta * \Omega_{1}+\delta * \Omega_{1}|A|^{2}
$$

In particular, $* \Omega_{1}$ has a uniform lower bound, each $\Sigma_{t}$ can be written as the graph of a map $f_{t}: \Sigma_{1} \rightarrow \Sigma_{2}$, and $f_{t}$ has uniform gradient bound.

Integrating $\frac{d}{d t} * \Omega_{1} \geq \Delta * \Omega_{1}+\delta * \Omega_{1}|A|^{2}$ over space and time from $t=0$ to $t=\infty$ and noting that $\int_{\Sigma_{t}} * \Omega_{1}$ is constant in $t$, we get

$$
\delta \int_{0}^{\infty} \int_{\Sigma_{t}} * \Omega_{1}|A|^{2} \leq \int_{0}^{\infty} \int_{\Sigma_{t}} * \Omega_{1}|H|^{2}
$$

For a mean curvature flow, $\int_{0}^{\infty} \int_{\Sigma_{t}}|H|^{2}<\infty$, thus $\int_{0}^{\infty} \int_{\Sigma_{t}}|A|^{2}<\infty$. We can extract a subsequence $t_{i} \rightarrow \infty$ such that $\int_{\Sigma_{t_{i}}}|A|^{2} \rightarrow 0$. Because each $f_{t_{i}}$ has bounded gradient, this is the same as $\int_{\Sigma_{1}}\left|\nabla d f_{t_{i}}\right|^{2} \rightarrow 0$. Therefore, $f_{t_{i}}$ is a bounded sequence in both $C^{\alpha}$ and $W^{2,2}$. By passing to a subsequence if necessary, there exists an $f_{\infty} \in \operatorname{Lip} \cap W^{2,2}$ such that $f_{t_{i}} \rightarrow f_{\infty}$ in $C^{\alpha}$ for any $\alpha<1$ and $f_{t_{i}} \rightarrow f_{\infty}$ weakly in $W^{2,2}$. As $d f_{t_{i}} \rightarrow d f_{\infty}$ weakly in $W^{1,2}$, by lower semicontinuity of the energy functional (see for example Theorem 1.8.1 in [8]), we have

$$
\int_{\Sigma_{1}}\left|\nabla d f_{\infty}\right|^{2} \leq \liminf \int_{\Sigma_{1}}\left|\nabla d f_{t_{i}}\right|^{2}=0
$$

By elliptic regularity, it is easy to see that $f_{\infty}$ is smooth and linear. $\Sigma_{\infty}$, the graph of $f_{\infty}$, is thus a totally geodesic (flat indeed) submanifold. Since $f_{t_{i}} \rightarrow f_{\infty}$ in $C^{\alpha}$ for any $\alpha<1$, we have the distance $d\left(\Sigma_{t_{i}}, \Sigma_{\infty}\right) \rightarrow 0$. The uniform convergence of $f_{t}$ follows as the proof of Theorem $\mathrm{C}$ in [15] where we use the property that distance function to any totally geodesic submanifold in a Riemannian manifold of non-positive sectional curvature is convex to show that $\max _{p \in \Sigma_{t}} d\left(p, \Sigma_{\infty}\right)$ is non-increasing in $t$ along the mean curvature flow.

Theorem B. Let $M=S^{n}\left(k_{1}\right) \times \Sigma_{2}^{m}$ be the product of an $n$-sphere of curvature $k_{1}>0$ and a two-dimensional compact Riemannian manifold $\Sigma_{2}$ of constant curvature $k_{2}$ and $k_{1} \geq\left|k_{2}\right|$. If the Gauss map of a compact oriented submanifold $\Sigma$ of $M$ lies in $\mathfrak{S}$, then along the mean curvature flow, the Gauss map of $\Sigma_{t}$ remains in $\mathfrak{S}$. The flow exists smoothly for all time and converges to a totally geodesic submanifold. 
Proof. The proof follows the same strategy as that of Theorem A. We actually show the image of the Gauss map never hits the boundary of $\mathfrak{S}$. Suppose the contrary happens at $t=t_{0}$. Again, we look at the equation of $* \Omega_{1}$. Using $\left|\lambda_{1} \lambda_{2}\right| \leq 1$ for $0 \leq t<t_{0}$ and Proposition 3.2 in [16], we see

$$
\begin{aligned}
\frac{d}{d t} * \Omega_{1} \geq & \Delta * \Omega_{1} \\
& +* \Omega_{1} \sum_{i} \frac{\lambda_{i}^{2}}{1+\lambda_{i}^{2}}\left[k_{1}\left(\sum_{j \neq i} \frac{2}{1+\lambda_{j}^{2}}\right)+k_{2}\left(1-n+\sum_{j \neq i} \frac{4}{1+\lambda_{j}^{2}}\right) .\right]
\end{aligned}
$$

The last term comes from the curvature of $M$. Rewrite

$$
\begin{aligned}
& k_{1}\left(\sum_{j \neq i} \frac{2}{1+\lambda_{j}^{2}}\right)+k_{2}\left(1-n+\sum_{j \neq i} \frac{2}{1+\lambda_{j}^{2}}\right) \\
& \quad=\frac{k_{1}-k_{2}}{2}(n-1)+\frac{k_{1}+k_{2}}{2}\left(\sum_{j \neq i} \frac{2}{1+\lambda_{j}^{2}}+1-n\right) .
\end{aligned}
$$

We claim the curvature term is always non-negative under our assumption. Since $k_{1}-k_{2} \geq 0, k_{1}+k_{2} \geq 0$, we only need to show

$$
\sum_{i=1}^{n} \frac{\lambda_{i}^{2}}{1+\lambda_{i}^{2}}\left(1-n+\sum_{k \neq i}^{n} \frac{4}{1+\lambda_{k}^{2}}\right) \geq 0
$$

This is indeed

$$
\frac{\lambda_{1}^{2}}{1+\lambda_{1}^{2}}\left(n-3+\frac{4}{1+\lambda_{2}^{2}}\right)+\frac{\lambda_{2}^{2}}{1+\lambda_{2}^{2}}\left(n-3+\frac{4}{1+\lambda_{1}^{2}}\right)
$$

This can be rewritten as

$$
\frac{(n+1) \lambda_{1}^{2}+(n+1) \lambda_{2}^{2}+2(n-3) \lambda_{1}^{2} \lambda_{2}^{2}}{\left(1+\lambda_{1}^{2}\right)\left(1+\lambda_{2}^{2}\right)}
$$

which is non-negative under the assumption $\left|\lambda_{1} \lambda_{2}\right| \leq 1$.

Therefore, at $t_{0}, \Sigma_{t_{0}}$ remains a graph and $\min _{\Sigma_{t_{0}}} \eta=0$. Take any $p$ so that $\eta\left(p, t_{0}\right)=0$. We may assume $\lambda_{1}>0, \lambda_{2}>0$ at $p$.

As before, we choose orthonormal basis at $p$ that corresponds to the singular value decomposition of $d f$. We can extend the orthonormal basis 
$\left\{a_{i}\right\}_{i=1 \cdots n}$ at $T_{\pi_{1}(p)} \Sigma_{1}$ to an orthonormal frame field in a neighborhood $U_{1} \subset$ $\Sigma_{1}$ such that at this point $p, \nabla^{M} a_{i}=0, i=1 \cdots n$. This is possible because the Riemannian structure is a product and each $\Sigma_{1}$ slice is totally geodesic in $M$. Take

$$
\Omega=\Omega_{1}-\Omega_{2} \wedge \omega
$$

where $\omega=a_{3}^{*} \wedge \cdots \wedge a_{n}^{*}$.

$\Omega$ is an $n$-form defined on $U=U_{1} \times \Sigma_{2}$ that satisfies $\nabla^{M} \Omega=0$ at $p$.

Now, we extend $\Omega$ to a global form on $M$. Take a cut-off function $\phi$ such that $\phi \equiv 1$ in a neighborhood of $p$ and $\phi$ has compact support. Then

$$
\Omega=\Omega_{1}-\phi \Omega_{2} \wedge \omega
$$

is such a global extension. Now, $* \Omega\left(p, t_{0}\right)=0$ and $\left(p, t_{0}\right), * \Omega>0$ for $0 \leq t<t_{0}$ and $* \Omega \geq 0$ at $t_{0}$. Therefore, $\frac{d}{d t} * \Omega \leq 0$ and $\Delta * \Omega \geq 0$ at $\left(p, t_{0}\right)$.

We recall the evolution equation from equation (3.6).

$$
\begin{aligned}
\frac{d}{d t} * \Omega= & \Delta * \Omega+* \Omega\left(\sum_{\alpha, i, k} h_{\alpha i k}^{2}\right) \\
& -2 \sum_{\alpha, \beta, k}\left[\Omega_{\alpha \beta 3 \cdots n} h_{\alpha 1 k} h_{\beta 2 k}+\Omega_{\alpha 2 \beta \cdots n} h_{\alpha 1 k} h_{\beta 3 k}\right. \\
& \left.+\cdots+\Omega_{1 \cdots(n-2) \alpha \beta} h_{\alpha(n-1) k} h_{\beta n k}\right] \\
& -\sum_{\alpha, k}\left[\Omega_{\alpha 2 \cdots n} R_{\alpha k k 1}+\cdots+\Omega_{1 \cdots(n-1) \alpha} R_{\alpha k k n}\right] \\
& +*\left(\nabla_{H}^{M} \Omega\right)-\left(\nabla_{e_{k}}^{M} \nabla_{e_{k}}^{M} \Omega\right)\left(e_{1}, \cdots, e_{n}\right) \\
& -2\left(\nabla_{e_{k}}^{M} \Omega\right)\left(e_{\alpha}, \cdots, e_{n}\right) h_{\alpha i_{1} k}-\cdots-2\left(\nabla_{e_{k}}^{M} \Omega\right)\left(e_{1}, \cdots, e_{\alpha}\right) h_{\alpha i_{n} k}
\end{aligned}
$$

By the way $\Omega$ is constructed, $\nabla^{M} \Omega=0$ at $p$. We claim the term $\left(\nabla_{e_{k}}^{M} \nabla_{e_{k}}^{M} \Omega\right)\left(e_{1}, \cdots, e_{n}\right)$ also vanishes at $p$.

In fact, consider

$$
\begin{aligned}
& \nabla_{e_{k}}^{M} \nabla_{e_{k}}^{M}\left(\Omega_{2} \wedge \omega\right)\left(e_{1}, \cdots, e_{n}\right) \\
& \quad=\left(\Omega_{2} \wedge \nabla_{e_{k}}^{M} \nabla_{e_{k}}^{M} \omega\right)\left(e_{1}, \cdots, e_{n}\right) \\
& \quad=\frac{\lambda_{1} \lambda_{2}}{\sqrt{\left(1+\lambda_{1}^{2}\right)\left(1+\lambda_{2}^{2}\right)}}\left(\nabla_{e_{k}}^{M} \nabla_{e_{k}}^{M} \omega\right)\left(a_{3}, \cdots, a_{n}\right)
\end{aligned}
$$

For $i=3, \cdots, n,\left(\nabla_{X}^{M} \nabla_{Y}^{M} a_{i}^{*}\right)\left(a_{i}\right)=X\left(\nabla_{Y}^{M} a_{i}^{*}\left(a_{i}\right)\right)-\left(\nabla_{Y}^{M} a_{i}^{*}\right)\left(\nabla_{X}^{M} a_{i}\right)$. $\left(\nabla_{Y}^{M} a_{i}^{*}\right)\left(a_{i}\right)=\left\langle\nabla_{Y}^{M} a_{i}, a_{i}\right\rangle=\left\langle\nabla_{Y}^{\Sigma_{1}} a_{i}, a_{i}\right\rangle$ is zero, so $X\left(\nabla_{Y}^{M} a_{i}^{*}\left(a_{i}\right)\right)=0$. 
Therefore, $\nabla_{X}^{M} \nabla_{Y}^{M} a_{i}^{*}\left(a_{i}\right)=0$ at $p$. Since $\omega=a_{3}^{*} \wedge \cdots \wedge a_{n}^{*}$, we get $\left(\nabla_{e_{k}}^{M} \nabla_{e_{k}}^{M} \omega\right)\left(a_{3}, \cdots, a_{n}\right)=0$.

Now, the left-hand side of equation (4.10) is non-positive, we shall show the curvature term

$$
-\sum_{\alpha, k}\left[\Omega_{\alpha 2 \cdots n} R_{\alpha k k 1}+\cdots+\Omega_{1 \cdots(n-1) \alpha} R_{\alpha k k n}\right]
$$

is strictly positive, thus achieves contradiction because all other terms on the right-hand side are non-negative.

We calculate the curvature term.

$$
\Omega\left(e_{\alpha}, e_{2}, \cdots, e_{n}\right)=-\delta_{\alpha, n+1} \frac{\lambda_{1}+\lambda_{2}}{\sqrt{\left(1+\lambda^{2}\right)\left(1+\lambda_{2}^{2}\right)}}
$$

Likewise,

$$
\Omega\left(e_{1}, e_{\alpha}, e_{3} \cdots, e_{n}\right)=-\delta_{\alpha, n+2} \frac{\lambda_{1}+\lambda_{2}}{\sqrt{\left(1+\lambda^{2}\right)\left(1+\lambda_{2}^{2}\right)}}
$$

and

$$
\Omega\left(e_{1}, e_{2}, e_{\alpha}, e_{4} \cdots, e_{n}\right)=0
$$

We assume $\Sigma_{1}$ and $\Sigma_{2}$ are of constant curvature $k_{1}$ and $k_{2}$ respectively, and by the calculation in [16], we have:

$$
\begin{aligned}
\sum_{k} R\left(e_{\alpha}, e_{k}, e_{k}, e_{i}\right) & \\
=k_{1} & {\left[\sum_{k}\left\langle\pi_{1}\left(e_{\alpha}\right), \pi_{1}\left(e_{k}\right)\right\rangle\left\langle\pi_{1}\left(e_{k}\right), \pi_{1}\left(e_{i}\right)\right\rangle\right.} \\
& \left.-\left\langle\pi_{1}\left(e_{\alpha}\right), \pi_{1}\left(e_{i}\right)\right\rangle \sum_{k}\left\langle\pi_{1}\left(e_{k}\right), \pi_{1}\left(e_{k}\right)\right\rangle\right] \\
& +k_{2}\left[\sum_{k}\left\langle\pi_{2}\left(e_{\alpha}\right), \pi_{2}\left(e_{k}\right)\right\rangle\left\langle\pi_{2}\left(e_{k}\right), \pi_{2}\left(e_{i}\right)\right\rangle\right. \\
& \left.-\left\langle\pi_{2}\left(e_{\alpha}\right), \pi_{2}\left(e_{i}\right)\right\rangle \sum_{k}\left\langle\pi_{2}\left(e_{k}\right), \pi_{2}\left(e_{k}\right)\right\rangle\right] \\
=k_{1} & {\left[\sum_{k}\left\langle\pi_{1}\left(e_{\alpha}\right), \pi_{1}\left(e_{k}\right)\right\rangle\left\langle\pi_{1}\left(e_{k}\right), \pi_{1}\left(e_{i}\right)\right\rangle\right.}
\end{aligned}
$$




$$
\begin{aligned}
& \left.-\left\langle\pi_{1}\left(e_{\alpha}\right), \pi_{1}\left(e_{i}\right)\right\rangle \sum_{k}\left|\pi_{1}\left(e_{k}\right)\right|^{2}\right] \\
& +k_{2}\left[(n-1)\left\langle\pi_{1}\left(e_{\alpha}\right), \pi_{1}\left(e_{i}\right)\right\rangle+\sum_{k}\left\langle\pi_{1}\left(e_{\alpha}\right), \pi_{1}\left(e_{k}\right)\right\rangle\left\langle\pi_{1}\left(e_{k}\right), \pi_{1}\left(e_{i}\right)\right\rangle\right. \\
& \left.-\left\langle\pi_{1}\left(e_{n+i}\right), \pi_{1}\left(e_{i}\right)\right\rangle \sum_{k}\left|\pi_{1}\left(e_{k}\right)\right|^{2}\right]
\end{aligned}
$$

Because $e_{n+i}=\frac{1}{\sqrt{1+\lambda_{i}^{2}}}\left(a_{n+i}-\lambda_{i} a_{i}\right), \pi_{1}\left(e_{n+i}\right)=\frac{-\lambda_{1}}{\sqrt{1+\lambda_{i}^{2}}} a_{i}$. Likewise, $\pi_{1}\left(e_{k}\right)=\frac{1}{\sqrt{1+\lambda_{k}^{2}}} a_{k}$.

Therefore,

$$
R_{n+i, k k i}=\frac{\lambda_{i}}{1+\lambda_{i}^{2}}\left[k_{1}\left(\sum_{k \neq i} \frac{1}{1+\lambda_{k}^{2}}\right)+k_{2}\left(1-n+\sum_{k \neq i} \frac{1}{1+\lambda_{k}^{2}}\right)\right]
$$

Therefore, the curvature term

$$
-\sum_{\alpha, k}\left[\Omega_{\alpha 2 \cdots n} R_{\alpha k k 1}+\cdots+\Omega_{1 \cdots(n-1) \alpha} R_{\alpha k k n}\right]
$$

in equation (3.6) becomes

$$
\begin{aligned}
& \frac{\left(\lambda_{1}+\lambda_{2}\right)}{\sqrt{\left(1+\lambda_{1}^{2}\right)\left(1+\lambda_{2}^{2}\right)}} \sum_{i=1}^{2} \frac{\lambda_{i}}{1+\lambda_{i}^{2}}\left[k_{1}\left(\sum_{j \neq i}^{n} \frac{1}{1+\lambda_{j}^{2}}\right)+k_{2}\left(1-n+\sum_{j \neq i}^{n} \frac{1}{1+\lambda_{j}^{2}}\right)\right] \\
& k_{1}\left(\sum_{j \neq i} \frac{1}{1+\lambda_{j}^{2}}\right)+k_{2}\left(1-n+\sum_{j \neq i} \frac{1}{1+\lambda_{j}^{2}}\right)=\frac{k_{1}-k_{2}}{2}(n-1) \\
& +\frac{k_{1}+k_{2}}{2}\left(\sum_{j \neq i} \frac{2}{1+\lambda_{j}^{2}}+1-n\right)
\end{aligned}
$$

Since $\lambda_{1}+\lambda_{2}>0$ and $k_{1}-k_{2} \geq 0, k_{1}+k_{2} \geq 0$, we only need to show

$$
\sum_{i=1}^{n} \frac{\lambda_{i}}{1+\lambda_{i}^{2}}\left(1-n+\sum_{k \neq i}^{n} \frac{2}{1+\lambda_{k}^{2}}\right) \geq 0
$$


This is indeed

$$
\frac{\lambda_{1}}{1+\lambda_{1}^{2}}\left(n-3+\frac{2}{1+\lambda_{2}^{2}}\right)+\frac{\lambda_{2}}{1+\lambda_{2}^{2}}\left(n-3+\frac{2}{1+\lambda_{1}^{2}}\right)
$$

This can be rewritten as

$$
(n-2) \frac{\left(\lambda_{1}+\lambda_{2}\right)\left(1+\lambda_{1} \lambda_{2}\right)}{\left(1+\lambda_{1}^{2}\right)\left(1+\lambda_{2}^{2}\right)}+\frac{\left(\lambda_{1}+\lambda_{2}\right)\left(1-\lambda_{1} \lambda_{2}\right)}{\left(1+\lambda_{1}^{2}\right)\left(1+\lambda_{2}^{2}\right)}
$$

which is strictly positive under the assumption $\left|\lambda_{1} \lambda_{2}\right|<1$.

Now, we turn to long-time existence and convergence. As in Theorem A, we can show

$$
\left|\lambda_{1} \lambda_{2}\right| \leq 1-\delta
$$

Recall the equation satisfied by $* \Omega_{1}$,

$$
\begin{aligned}
\frac{d}{d t} & * \Omega_{1} \geq \Delta * \Omega_{1}+\delta * \Omega_{1}|A|^{2} \\
& +* \Omega_{1}\left\{\frac{k_{1}-k_{2}}{2}(n-1)\left[\sum_{i} \frac{\lambda_{i}^{2}}{1+\lambda_{i}^{2}}\right]+\frac{k_{1}+k_{2}}{2}\left[(n-2) \frac{\lambda_{1}^{2}+\lambda_{2}^{2}+2 \lambda_{1}^{2} \lambda_{2}^{2}}{\left(1+\lambda_{1}^{2}\right)\left(1+\lambda_{2}^{2}\right)}\right.\right. \\
& \left.\left.+\frac{\lambda_{1}^{2}+\lambda_{2}^{2}-2 \lambda_{1}^{2} \lambda_{2}^{2}}{\left(1+\lambda_{1}^{2}\right)\left(1+\lambda_{2}^{2}\right)}\right]\right\}
\end{aligned}
$$

In fact, $\sum_{i} \frac{\lambda_{i}^{2}}{1+\lambda_{i}^{2}}=\frac{\lambda_{1}^{2}+\lambda_{2}^{2}+2 \lambda_{1}^{2} \lambda_{2}^{2}}{\left(1+\lambda_{1}^{2}\right)\left(1+\lambda_{2}^{2}\right)}$. Since $* \Omega_{1}=\frac{1}{\sqrt{\left(1+\lambda_{1}^{2}\right)\left(1+\lambda_{2}^{2}\right)}}$, we have $1-* \Omega_{1}^{2}=\frac{\lambda_{1}^{2}+\lambda_{2}^{2}+\lambda_{1}^{2} \lambda_{2}^{2}}{\left(1+\lambda_{1}^{2}\right)\left(1+\lambda_{2}^{2}\right)}$. It is not hard to see that there exists a constant $c^{\prime}$ such that $\lambda_{1}^{2}+\lambda_{2}^{2}-2 \lambda_{1}^{2} \lambda_{2}^{2} \geq c^{\prime}\left(\lambda_{1}^{2}+\lambda_{2}^{2}+\lambda_{1}^{2} \lambda_{2}^{2}\right)$ if $\left|\lambda_{1} \lambda_{2}\right| \leq 1-\delta$.

Therefore,

$$
\frac{d}{d t} * \Omega_{1} \geq \Delta * \Omega_{1}+\delta * \Omega_{1}|A|^{2}+c * \Omega_{1}\left(1-* \Omega_{1}^{2}\right)
$$

for some constant $c>0$.

As in the proof of Theorem A in [16], this equation implies long time existence by blowing up argument and White's regularity Theorem [17].

By maximum principle, $\min _{\Sigma_{t}} * \Omega_{1} \rightarrow 1$ as $t \rightarrow \infty$, then we can use the estimate in the proof of Theorem B in [16] to show $\max _{\Sigma_{t}}|A|^{2} \rightarrow 0$ and apply Simon's general convergence theorem [9]. We get smooth convergence in this case. In the limit, $* \Omega_{1}=1$ and thus $\lambda_{1}=\lambda_{2}=0$. 
Corollary. If $f$ is any smooth map from $S^{n}$ to $S^{2}$ and if at each point, the restriction of $d f$ to any two dimensional subspace is area decreasing, then $f$ is homotopic to a constant map along the mean curvature flow.

Again, we remark the condition is equivalent to the comass of $f^{*} \Omega_{2}$ is less than one, where $\Omega_{2}$ is the area form on $S^{2}$.

Remark 4.1. This paper was finished in January 2002. In the past few years, there were several more long-time existence results of higher codimension mean curvature flows. For example, the work of Chen-Li-Tian [1], Smoczyk [10], [11] and Smoczyk-Wang[12]. In particular, the results of this paper were generalized to allow the dimension of the target manifold to be arbitrary in Tsui-Wang [13].

\section{References.}

[1] J. Y. Chen, J. Y. Li, and G. Tian, Two-dimensional graphs moving by mean curvature flow. Acta Math. Sin. (Engl. Ser.) 18 (2) (2002), 209-224.

[2] K. Ecker and G. Huisken, Mean curvature evolution of entire graphs. Ann. of Math. (2) 130 (3) (1989), 453-471.

[3] K. Ecker and G. Huisken, Interior estimates for hypersurfaces moving by mean curvature. Invent. Math. 105 (3) (1991), 547-569.

[4] H. Federer, Geometric measure theory. Die Grundlehren der mathematischen Wissenschaften, Band 153 Springer-Verlag New York Inc., New York 1969.

[5] R. Hamilton, Four-manifolds with positive curvature operator. J. Differential Geom. 24 (2) (1986), 153-179.

[6] R. Harvey and H. B. Lawson, Calibrated geometries. Acta Math. 148 (1982), 47-157.

[7] G. Huisken, Asymptotic behavior for singularities of the mean curvature flow. J. Differential Geom. 31 (1) (1990), 285-299.

[8] C. B. Morrey, Jr. Multiple integrals in the calculus of variations. Die Grundlehren der mathematischen Wissenschaften, Band 130 SpringerVerlag New York, Inc., New York 1966. 
[9] L. Simon, Asymptotics for a class of non-linear evolution equations, with applications to geometric problems. Ann. of Math. (2) 118 (3) (1983), 525-571.

[10] K. Smoczyk, Angle theorems for the Lagrangian mean curvature flow. Math. Z. 240 (4) (2002), 849-883.

[11] K. Smoczyk, Longtime existence of the Lagrangian mean curvature flow. Calc. Var. Partial Differential Equations 20 (1) (2004), 25-46.

[12] K. Smoczyk and M.-T. Wang, Mean curvature flows of Lagrangians submanifolds with convex potentials. J. Differential Geom. 62 (2) (2002), 243-257.

[13] M.-P. Tsui and M.-T. Wang, Mean curvature flows and isotopy of maps between spheres. Comm. Pure Appl. Math. 57 (8) (2004), 1110-1126.

[14] M.-T. Wang, Mean curvature flow of surfaces in Einstein fourmanifolds. J. Differential Geom. 57 (2) (2001), 301-338.

[15] M.-T. Wang, Deforming area preserving diffeomorphism of surfaces by mean curvature flow. Math. Res. Lett. 8 (5-6) (2001), 651-661.

[16] M.-T. Wang, Long-time existence and convergence of graphic mean curvature flow in arbitrary codimension. Invent. Math. 148 (3) (2002), $525-543$.

[17] B. White, A local regularity theorem for classical mean curvature flow. preprint, 2000.

Available at http://math.stanford.edu/ white/preprint.htm

RECEIVED JANUARY 10, 2002. 